\title{
ON THE COMPACTNESS OF THE SPACE $L_{p}$
}

\author{
BY J. D. TAMARKIN*
}

1. Introduction. Let $R_{n}$ be the $n$-dimensional euclidean space and $L_{p}(p>1)$ the function-space consisting of all the functions $f(x)$ defined and measurable over $R_{n}$, and such that the integrals

$$
\int_{R_{n}}|f(x)|^{p} d x<\infty,
$$

while the metric of the space $L_{p}$ is defined, as usual, by the "distance"

$$
\|f\| \equiv\left[\int_{R_{n}}|f(x)|^{p} d x\right]^{1 / p} .
$$

All the notions of boundedness, convergence, limits, approximations, etc., used in this note will be relative to this metric, unless explicitly specified to the contrary.

Let $S(x, \epsilon)$ be the $n$-dimensional sphere with center at $x$ and radius $\epsilon$. We designate by $V(\epsilon)$ the volume of $S(x, \epsilon)$, and by

$$
f_{\epsilon}(x) \equiv \frac{1}{V(\epsilon)} \int_{S(x, \epsilon)} f(y) d y
$$

the moving average of $f(x)$. Finally, let $C_{N}$ be the $n$-dimensional cube, with center at the origin and edges of length $2 N$ parallel to the coordinate axes. We set

$$
f^{N}(x)=\left\{\begin{array}{l}
f(x) \text { when } x \text { is in } C_{N}, \\
0 \text { elsewhere. }
\end{array}\right.
$$

A set $\mathfrak{F}$ of elements of $L_{p}$ is called compact if every subset of $\mathfrak{F}$ contains at least one convergent sequence. In a recent paper Kolmogoroff $\dagger$ has derived necessary and sufficient conditions in order that $\mathfrak{F}$ be compact, under the restriction that all the elements of $\mathfrak{F}$ vanish outside of a fixed bounded measurable point

* Presented to the Society, October 31, 1931.

$\dagger$ Göttinger Nachrichten, 1931, pp. 60-63. 
set $F$ of $R_{n}$. In order that a set $\mathfrak{F}$ of this type be compact it is necessary and sufficient that there exist a fixed positive constant $K$ and, for each given positive $\delta$, a positive $\epsilon=\epsilon(\delta)$ depending only on $\delta$ and $\mathfrak{F}$, such that for all elements of $\mathfrak{F}$,

$$
\begin{aligned}
& \int_{F}|f(x)|^{p} d x \leqq K \\
& \int_{F}\left|f(x)-f_{\epsilon}(x)\right|^{p} d x \leqq \delta .
\end{aligned}
$$

In the case where $F$ is not bounded, in particular where $F=R_{n}$, the conditions above, being still necessary, are no longer sufficient for the compactness of $\mathfrak{F}$. This can be shown by the following simple example $(n=1)$. Let

$$
\mathfrak{F} \equiv\left\{f_{\nu}(x)\right\}, \quad(\nu=0, \pm 1, \pm 2, \cdots),
$$

where $f_{\nu}(x)=1$ for $\nu \leqq x<\nu+1$, and 0 elsewhere. It is plain that $\left\|f_{i}-f_{j}\right\|=2^{1 / p}, i \neq j$, so that no subsequence of $\mathfrak{F}$ can converge. On the other hand we have, for all values of $\nu$,

$$
\begin{aligned}
\left\|f_{\nu}\right\| & =1 \\
f_{\nu}(x)=f_{0}(x-\nu) & =\frac{1}{2 \epsilon} \int_{x-\nu-\epsilon}^{x-\nu+\epsilon}\left[f_{0}(x-\nu)-f_{0}(\xi)\right] d \xi \\
\left\|f_{\nu}-\left(f_{\nu}\right)_{\epsilon}\right\|^{p} & =\int_{-\infty}^{\infty} d x\left|\frac{1}{2 \epsilon} \int_{x-\nu-\epsilon}^{x-\nu+\epsilon}\left[f_{0}(x-\nu)-f_{0}(\xi)\right] d \xi\right|^{p} \\
& =\left\|f_{0}-\left(f_{0}\right)_{\epsilon}\right\|^{p}
\end{aligned}
$$

and so the distance $\left\|f_{\nu}-\left(f_{\nu}\right)_{\epsilon}\right\|$ can be made arbitrarily small by taking $\epsilon$ sufficiently small, uniformly in $\nu$. Hence the conditions above are satisfied but $\mathfrak{F}$ is not compact. The purpose of the present note is to derive a set of necessary and sufficient conditions for the compactness of any set $\mathfrak{F} \subset L_{p}$. This can be easily done by introducing some slight modifications in Kolmogoroff's arguments.

2. Conditions for Compactness. The conditions for compactness of an arbitrary set $\mathfrak{F} \subset L_{p}$ are given by the following theorem. 
THEOREM. In order that a set $\mathfrak{F} \subset L_{p}(p>1)$ be compact it is necessary and sufficient that $\mathfrak{F}$ be bounded, and that the elements $f(x)$ of $\mathfrak{F}$ should be approximated, arbitrarily closely and uniformly over $\mathfrak{F}$, by the corresponding functions $f_{\mathrm{e}}$ and $f^{N}$. In other words, there should exist a constant $F_{0} \equiv F_{0}(\mathfrak{F})$, and, for a given positive $\delta$, two constants, $\epsilon \equiv \epsilon(\delta ; \mathfrak{F})$ and $N_{0} \equiv N_{0}(\delta ; \mathfrak{F})$, depending only on $\mathfrak{F}$, or on $\delta$ and $\mathfrak{F}$ respectively, but not on $f(x) \subset \mathfrak{F}$, such that the following conditions are satisfied:

$$
\begin{aligned}
\|f\| & \leqq F_{0} ; \\
\left\|f-f_{h}\right\| & \leqq \delta, 0<h \leqq \epsilon ; \\
\left\|f-f^{N}\right\| & \leqq \delta, N \geqq N_{0} ;
\end{aligned}
$$

for all elements $f \subset \mathfrak{F}$.

The proof of this theorem is based upon several simple lemmas which are mostly well known and are stated here merely for the convenience of the reader.

Lemma 1. If $f, g \subset L_{p}(p \geqq 1)$, then

$$
\begin{aligned}
\left\|f_{h}-g_{h}\right\| & \leqq\|f-g\|, \\
\left\|f^{N}-g^{N}\right\| & \leqq\|f-g\| .
\end{aligned}
$$

In particular,

$$
\left\|f_{h}\right\| \leqq\|f\|,\left\|f^{N}\right\| \leqq\|f\| .
$$

Lemma 2. For a fixed element $f \subset L_{p}(p \geqq 1)$,

$$
\begin{aligned}
& \left\|f-f_{h}\right\| \rightarrow 0 \text { as } \quad h \rightarrow 0, \\
& \left\|f-f^{N}\right\| \rightarrow 0 \text { as } \quad N \rightarrow \infty .
\end{aligned}
$$

LeMma 3. Under the assumption of Lemma 2,

$$
\left\|f^{N}-\left(f^{N}\right)_{h}\right\| \leqq 2\left\|f-f^{N}\right\|+\left\|f-f_{h}\right\| .
$$

To simplify the notation we shall consider only the onedimensional case, so that $-\infty<x<\infty$ and

$$
\|f\| \equiv\left[\int_{-\infty}^{\infty}|f(x)|^{p} d x\right]^{1 / p} .
$$


The definition of $f_{h}(x)$ will be slightly modified by setting

$$
f_{h}(x) \equiv h^{-1} \int_{x}^{x+h} f(t) d t=h^{-1} \int_{0}^{h} f(x+t) d t
$$

while, as before,

$$
f^{N}(x)=f(x), \text { or } 0, \text { according as }|x| \leqq N, \text { or }>N .
$$

The proofs of the lemmas above will be only sketched.

Formula (7) follows directly from the definition of $f^{N}(x)$, $g^{N}(x)$. Formula (6) is derived by using Hölder's inequality,

$$
\begin{aligned}
& \quad\left|f_{h}(x)-g_{h}(x)\right|^{p} \leqq h^{-1} \int_{0}^{h}|f(x+t)-g(x+t)|^{p} d t, \\
& \int_{-\infty}^{\infty}\left|f_{h}(x)-g_{h}(x)\right|^{p} d x \\
& \quad \leqq h^{-1} \int_{0}^{h} d t \int_{-\infty}^{\infty}|f(x+t)-g(x+t)|^{p} d x=\|f-g\|^{p},
\end{aligned}
$$

when $p>1$, and even more simply when $p=1$. Formula $(10)$ is obvious, while (9) follows from

$$
\begin{aligned}
\left|f(x)-f_{h}(x)\right|^{p} & \leqq h^{-p}\left[\int_{0}^{h}|f(x)-f(x+t)| d t\right]^{p} \\
& \leqq h^{-1} \int_{0}^{h}|f(x)-f(x+t)|^{p} d t
\end{aligned}
$$

and from a known theorem* according to which

$$
\int_{-\infty}^{\infty}|f(x)-f(x+t)|^{p} d x \rightarrow 0 \text { as } t \rightarrow 0, f \subset L_{p} .
$$

To prove (11) we observe that, by (6),

$$
\begin{aligned}
\left\|f^{N}-\left(f^{N}\right)_{h}\right\| & \leqq\left\|f^{N}-f\right\|+\left\|f-f_{h}\right\|+\left\|f_{h}-\left(f^{N}\right)_{h}\right\| \\
& \leqq 2\left\|f-f^{N}\right\|+\left\|f-f_{h}\right\| .
\end{aligned}
$$

* Hobson, Theory of Functions of a Real Variable, vol. I, 3d ed., 1927, pp. 636-639. The result is stated and proved there only for $p=1$ and $p=2$, but can be extended readily to the general case of any $p \geqq 1$. 
We now turn to the proof of our theorem. The necessity of conditions (i) and (ii) is established in precisely the same fashion as in the case of Kolmogoroff. To prove the necessity of (iii) we shall use an analogous argument. If (iii) is not satisfied, we can find a fixed positive $\delta$ and two sequences, of functions $\left\{f_{n}(x)\right\} \subset \mathfrak{F}$, and of positive numbers $\left\{N_{n}\right\} \rightarrow \infty$ as $n \rightarrow \infty$, such that, on setting

$$
f_{n}^{\prime} \equiv\left(f_{n}\right)^{N_{n}}
$$

we would have

$$
\left\|f_{n}-f_{n}^{\prime}\right\|>\delta \text {. }
$$

Hence, no matter what is $f(x) \subset L_{p}$, by (7),

$$
\begin{aligned}
\delta<\left\|f_{n}-f_{n}^{\prime}\right\| & \leqq\left\|f_{n}-f\right\|+\left\|f-f^{N_{n}}\right\|+\left\|f^{N_{n}}-f_{n}^{\prime}\right\| \\
& \leqq 2\left\|f-f_{n}\right\|+\left\|f-f^{N_{n}}\right\| .
\end{aligned}
$$

On the other hand, since $N_{n} \rightarrow \infty$, we have by (10)

$$
\left\|f-f^{N_{n}}\right\| \rightarrow \infty \text {. }
$$

The conclusion is that

$$
\lim _{n \rightarrow \infty}\left\|f-f_{n}\right\| \geqq \delta / 2,
$$

and so, no subsequence of $\left\{f_{n}(x)\right\}$ can converge. This shows that $\mathfrak{F}$ is not compact.

We pass on to the proof of the sufficiency of the conditions (i)-(iii). Assume that these conditions are satisfied. Let $\left\{f_{n}(x)\right\}$ be an arbitrary sequence of $\mathfrak{F}$. To abbreviate we put

$$
f_{n}(x) \equiv \phi(x), \quad f_{m}(x) \equiv \psi(x),
$$

where $n$ and $m$ are arbitrary positive integers. According to (ii) and (iii) a positive $h$ can be fixed so small and a positive $N$ so large that, for a given $\delta>0$,

$$
\left\|\phi-\phi^{N}\right\|, \quad\left\|\psi-\psi^{N}\right\|, \quad\left\|\phi-\phi_{h}\right\|, \quad\left\|\psi-\psi_{h}\right\| \leqq \delta .
$$

By (i), (8), and (11),

$$
\left\|\phi^{N}\right\| \leqq F_{0}, \quad\left\|\phi^{N}-\left(\phi^{N}\right)_{h}\right\| \leqq 3 \delta,
$$

whence, according to the result of Kolmogoroff, the set $\{\phi\}$ is compact. Hence a sequence of subscripts $\nu_{s}$ can be selected in 
such a way that when $n$ and $m \rightarrow \infty$ assuming the values belonging to this sequence, then

$$
\left\|\left(\phi^{N}\right)_{h}-\left(\psi^{N}\right)_{h}\right\| \rightarrow 0 .
$$

In particular, for $n$ and $m$ sufficiently large,

$$
\left\|\left(\phi^{N}\right)_{h}-\left(\psi^{N}\right)_{h}\right\| \leqq \delta .
$$

On combining these inequalities we get, with reference to (11), $\left\|f_{n}-f_{m}\right\| \leqq\left\|\phi-\phi^{N}\right\|+\left\|\phi^{N}-\left(\phi^{N}\right)_{h}\right\|$

$$
+\left\|\left(\phi^{N}\right)_{h}-\left(\psi^{N}\right)_{h}\right\|+\left\|\left(\psi^{N}\right)_{h}-\psi^{N}\right\|+\left\|\psi^{N}-\psi\right\| \leqq 9 \delta .
$$

This shows that an arbitrary sequence of $\mathfrak{F}$ contains a convergent subsequence, hence $\mathfrak{F}$ is compact.

3. Conclusion. It is interesting to observe that the conditions (i)-(iii) of our theorem are independent, in the sense that no two of them imply the third.

(a) That conditions (i) and (ii) do not imply (iii) is shown by the example of the set (5) which satisfies (i) and (ii) but not (iii), for

$$
\left\|f_{n}-\left(f_{n}\right)^{N}\right\|=1, \quad(0<N<n) .
$$

(b) That (i) and (iii) do not imply (ii) can be shown by the example of any set of functions which vanish outside of a fixed finite interval $(a, b)$, while the set is orthonormal for this interval.

(c) To prove that (ii) and (iii) do not imply (i) it suffices to consider the set $\left[f_{1}, f_{2}, \cdots, f_{n}, \cdots\right]$ defined by the condition that $f_{n}(x)=f(x)+n$ on $(0,1)$, and 0 elsewhere, $f(x)$ being an arbitrary function $\subset L_{p}$ and vanishing outside of $(0,1)$.

BROWN UNIVERSITY 